\title{
Statistical analysis of the flux of micrometeoroids at Mercury from both cometary and asteroidal components
}

\author{
P. Borin ${ }^{1}$, G. Cremonese ${ }^{1}$, and F. Marzari ${ }^{2}$ \\ 1 INAF-Astronomical Observatory of Padova, Vicolo dell'Osservatorio 5, 35131 Padova, Italy \\ e-mail: [patrizia.borin; gabriele.cremonese]@oapd.inaf.it \\ 2 Department of Physics and Astronomy, University of Padova, via Marzolo 8, 35131 Padova, Italy \\ e-mail: marzari@pd.infn.it
}

Received 17 June 2015 / Accepted 21 October 2015

\begin{abstract}
Context. Meteoroid impacts are an important source of neutral atoms for the exosphere of Mercury. We previously estimated the contribution of meteoroids originating in the asteroid belt for vapor release. In this paper, we concentrate on the cometary component of particles impacting the planet. Comets and asteroids are considered to be the two major sources of interplanetary dust particles in the solar system. The debate about which source contributes most to dust populating the solar system is still ongoing.

Aims. In this work, we compute the orbital evolution of dust particles produced by Jupiter-family comets (JFC) via $N$-body numerical integrations. From our numerical simulations, we compute the fraction of particles hitting Earth and Mercury's surface and the corresponding distribution of impact velocities. According to some authors more than $80 \%$ of all the incoming mass of meteoroids entering the Earth's atmosphere is concentrated in the mass range $10^{-7}-10^{-3} \mathrm{~g}$. In our model, we considered a slightly different range, $10^{-9}$ to $10^{-6} \mathrm{~g}$, to include possible uncertainty.

Methods. The orbital evolution of dust particles of different sizes is computed with a numerical integration code, which includes the effects of Poynting-Robertson drag, solar wind drag, and planetary perturbations.

Results. By comparing the impact frequency of grains evolving either from main belt asteroids or JFC we find that the cometary component is significantly less efficient in releasing dust particles on Mercury than on the Earth. The opposite occurs in the case of dust coming from the main belt with a flux higher at Mercury than on the Earth. This is mostly due to the different dynamical histories of the grains from their release until impact. This may have important implications for the vapor production rate on Mercury. We compare our results with previous estimates given by different authors.
\end{abstract}

Key words. methods: statistical - meteorites, meteors, meteoroids - comets: general - planets and satellites: individual: Mercury

\section{Introduction}

Comets and asteroids are considered to be the two major sources of solar system interplanetary dust particles (IDP). There has been a considerable debate whether comets or asteroids provide the greatest amount of dust in the solar system (see Dermott et al. 1984, 2002; Sykes et al. 2004; Fulle 2004). The relative proportions of asteroidal and cometary component in the zodiacal cloud is still an open issue. The IDP abundances are given by zodiacal light measurements, by meteoroid impacts onto spacecraft dust sensors (Mann \& Grün 1995; Mann et al. 1996; Mann 2004), and by samples collected by high altitude aircraft in the stratosphere (Love \& Brownlee 1993; Zolesky et al. 1994; Flynn 2002). Dust particles originating from comets generally have much higher Earth approaching and atmospheric entry velocities than those of asteroidal grains, but the number of Earth-crossing comets per year are considerably fewer than near-Earth asteroids (Weissman 2007).

The total cometary impact rate on Earth, based on observed comets is $1.4 \times 10^{-7}$ per year, or one impact every 7.2 Myr. About $48 \%$ of this total is due to Jupiter-family comets (JFC), $14 \%$ due to Halley-type comets, and $38 \%$ due to long-period comets, although half of the long-period comets arrive in brief cometary showers of 2-3 Myr duration (Weissman 2007). If one takes into account the impact rate estimates for long period comets, JFC and Encke type comets, then the total impact rate is
$1.1 \times 10^{-7}$ per year, or one impact every 9.1 Myr. If comet showers are included, these numbers increase to $1.4 \times 10^{-7}$ per year, or one impact every 7.2 Myr. These represent only a few percent of the impact rates of near-Earth asteroids (Weissman 2007). Also, the orbital integration of JFC, asteroidal and cometary dust particles under the gravitational influence of the planets, the Poynting-Robertson drag, radiation pressure, and solar wind drag support the previous estimates (Ipatov \& Mather 2005, 2006). These integrations demonstrated that by varying the ratio between the radiation pressure force and the gravitational force, the collision probabilities of cometary dust particles are lower than asteroidal dust particles. This is the case except for dust particles coming from comets, such as 10P/Tempel 2, which are closer to those particles coming from asteroids (Ipatov \& Mather 2005).

The production of dust by comets can be deduced from observations of dust tails and coma. Variation of cometary activity and uncertainties in determining the size distribution of ejected particles make it difficult to estimate the total mass production of a single comet (A'Hearn et al. 1995; Fernández 2009). There is a significant difference in the dynamics of dust particles produced by comets and asteroids (Jackson \& Zook 1992). Grains produced by asteroidal collisions have initial orbital elements that are similar to those of the parent body and then reflect the orbital distribution of the asteroid belt with low values of both 
eccentricity and inclination. After being generated in the asteroid belt, this dust evolves towards the Sun under the PoyntingRobertson drag, which further circularises the orbits that also reduce the inclination (Burns et al. 1979; Wyatt \& Whipple 1950; Sykes \& Greenberg 1986). Dust coming from comets is expected to move on trajectories with much higher eccentricity and inclination (Jackson \& Zook 1992). Since comets are much fewer in number than asteroids, tracing their current orbits would produce a relatively lumpy cloud. Whether a cometary component could be smooth depends on the outcome of the race between differential precession of the ejected cometary particle orbits, collisional lifetimes, and orbital decay timescales (Burns et al. 1979).

We study the long-term evolution of dust grains (i.e. $r<$ $1 \mathrm{~mm}$ ) originating from short period comets to Mercury as a completion of the previous work on dust particles coming from the main belt asteroid (Borin et al. 2009). By means of numerical simulations, we estimate the flux of dust particles on the surface of Mercury and their impact velocity distribution. We focus on dust particles produced by JFC, since Nesvorny et al. (2010) claim that the $85 \%-95 \%$ of the observed mid-infrared emission is produced by particles from JFCs and a quantity less than $10 \%$ by dust from long-period comets.

\section{Dynamical evolution model}

To estimate the meteoritic flux at the heliocentric distance of Mercury, we use the dynamical evolution model of dust particles of Marzari \& Vanzani (1994). This model numerically integrates a $(N+1)+M$ body problem (Sun $+N$ planets $+M$ body with negligible mass) with the high-precision integrator RA15. The initial orbital elements of all planets are taken from the JPL Horizon site. Radiation and solar wind pressure and Poynting-Robertson drag are included as perturbative forces together with the gravitational attractions of all the planets in the solar system.

The gravitational term is given by:

$\boldsymbol{F}_{\text {gra }}=\boldsymbol{F}^{\mathrm{k}}+\boldsymbol{F}^{\mathrm{d}}+\boldsymbol{F}^{\mathrm{ind}}$

where $\boldsymbol{F}^{\mathrm{k}}$ is the Keplerian force, $\boldsymbol{F}^{\mathrm{d}}$ is the direct force, and $\boldsymbol{F}^{\text {ind }}$ is the indirect force. Equation (1) can be written as

$$
\begin{aligned}
\boldsymbol{F}_{\text {gra }}= & \frac{G m\left(M_{\text {Sun }}+m\right) \boldsymbol{r}_{\text {Sun }}}{r_{\text {Sun }}^{3}} \\
& +\frac{G m \sum_{j=1}^{N} m_{j} \boldsymbol{r}_{j}}{r_{j}^{3}}+\frac{G m \sum_{j=1}^{N} m_{j} \boldsymbol{r}_{\text {Sun }, j}}{r_{\text {Sun }, j}^{3}},
\end{aligned}
$$

where $r_{\text {Sun }}$ is the distance between the Sun and dust particles, $r_{j}$ is the distance between planets and dust particles, $m$ is the mass of dust particles, and $N$ is the number of planets.

The non-gravitational term is made up of two terms: the radiation force, $\boldsymbol{F}_{\text {rad }}$ and the force given by the solar wind, $\boldsymbol{F}_{\text {wnd }}$,

$$
\begin{aligned}
\boldsymbol{F}_{\mathrm{ngra}} & =\boldsymbol{F}_{\mathrm{rad}}+\boldsymbol{F}_{\mathrm{wnd}} \\
& =\frac{S}{c}\left(1-\frac{\dot{r}}{c}\right) A Q_{\mathrm{pr}} \hat{\boldsymbol{p}}+\sum_{j} \frac{\eta_{j} u^{2}}{2} A C_{D, j} \hat{\boldsymbol{u}}=f_{r} \hat{\boldsymbol{p}}+f_{w} \hat{\boldsymbol{u}} .
\end{aligned}
$$

In the previous equations $\hat{\boldsymbol{p}}=\frac{\boldsymbol{c}-\boldsymbol{v}}{c}$, where $\boldsymbol{c}$ is the velocity of the light (anti-solar direction) and $v$ is the orbital velocity of the dust particle; $\hat{\boldsymbol{u}}=\frac{u}{u}$ with $\boldsymbol{u}=\boldsymbol{w}-\boldsymbol{v}$, where $\boldsymbol{w}$ is the solar wind flow bulk velocity in the average phase (Mukai et al. 1982; Marzari $\&$ Vanzani 1994). The parameter $\eta_{j}=n_{j} m_{j}$ is the spatial mass density of the component $j$ of the solar wind flow, with mass $m_{j}$ and number density $n_{j} ; A$ is the geometrical cross section of the grain; $Q_{\mathrm{pr}}$ is the dimensionless radiation-pressure coefficient averaged over the solar spectrum, and $C_{D, j}$ is the dimensionless drag coefficient due to the $\mathrm{j}$ component of the wind flow. The parameter $S$ is the solar radiation flux density at heliocentric distance $r$, and we can write $S=S_{0}\left(\frac{r_{0}}{r}\right)^{2}, w_{0} \simeq 4 \times 10^{2} \mathrm{~km} \mathrm{~s}^{-1}$ for $w$ at $1 \mathrm{AU}$, and $\eta_{p, 0}+\eta_{\alpha, 0} \simeq 1.2 \eta_{p, 0}$ (Marzari \& Vanzani 1994). The parameters $f_{r}$ and $f_{w}$ are the magnitude of the radiation force and solar wind force, respectively.

The efficiency of the radiation and corpuscular resistive forces can be expressed by defining their ratio to the solar gravity, i.e.,

$\beta_{r}=\frac{f_{r}}{f_{g}}\left[\frac{c}{c-\dot{r}}\right]=\left(\frac{S A Q_{\mathrm{pr}}}{c}\right) \cdot\left(\frac{G M_{\odot} m}{r^{2}}\right)^{-1}$,

and

$\beta_{w}=\frac{f_{w}}{f_{g}}\left[\frac{w}{|\boldsymbol{w}-\boldsymbol{v}|}\right]=\left(\frac{f_{w 0} \psi}{\kappa}\right) \cdot\left(\frac{G M_{\odot} m}{r^{2}}\right)^{-1}$,

with $\kappa=\frac{u}{w}$ and $\psi=\frac{f_{w}}{f_{w 0}}$, where $f_{w 0}$ is obtained from $f_{w}$ in the limit of neglecting the velocity dispersion of wind particles and taking no notice of the contribution of momentum carried away by the sputtered molecules to $\boldsymbol{F}_{\text {wnd }}$. The parameter $f_{g}$ is the magnitude of the gravitational force.

Assuming the reference-distance $r_{0}$ equal to $1 \mathrm{AU}$ and the dust particle with a spherical shape of radius $s$, we obtain

$\beta_{r}=\frac{3 S_{0} r_{0}^{2}}{4 G M_{\odot} c} \frac{Q_{\mathrm{pr}}}{\varrho S}=5.74 \times 10^{-5} \frac{Q_{\mathrm{pr}}}{\varrho s}$,

and

$\beta_{w}=\frac{3\left(\eta_{p, 0}+\eta_{\alpha, 0}\right) r_{0}^{2} w_{0}^{2}}{4 G M_{\odot}} \frac{\psi \kappa}{\varrho s} \simeq 3.27 \times 10^{-8} \frac{\psi \kappa}{\varrho s}$,

where $\varrho$ is the mass density of the dust particle measured, like $s$, in cgs units. The parameter $S$ is the solar radiation flux density at heliocentric distance $r$, and we can write $S=S_{0}\left(\frac{r_{0}}{r}\right)^{2}, w_{0} \simeq$ $4 \times 10^{7} \mathrm{~cm} / \mathrm{s}$ for $w$ at $1 \mathrm{AU}$, and $\eta_{p, 0}+\eta_{\alpha, 0} \simeq 1.2 \eta_{p, 0}$ (Marzari \& Vanzani 1994).

Finally, the relative importance of the radiation and corpuscular forces can be estimated by the parameter

$\gamma=\frac{\beta_{w}}{\beta_{r}} \simeq 5.7 \times 10^{-4} \frac{\psi \kappa}{Q_{\mathrm{pr}}}$.

In terms of the parameters $\beta_{r}$ and $\gamma$ the sum of the radiation and solar resistive forces takes the form

$$
\begin{aligned}
\boldsymbol{F}_{\text {ngra }}= & \beta_{r} f_{g}[(1+\gamma \cos \varphi) \hat{\boldsymbol{r}} \mp \gamma(\sin \varphi) \hat{\boldsymbol{\vartheta}}] \\
& -\beta_{r} f_{g}\left[\left(2+\gamma \frac{c}{w}\right) \frac{\dot{r}}{c} \hat{\boldsymbol{r}}+\left(1+\gamma \frac{c}{w}\right) \frac{r \dot{\vartheta}}{c} \hat{\boldsymbol{\vartheta}}\right],
\end{aligned}
$$

where the terms dependent and independent on a dust grain's velocity appear separated. Here $\hat{\boldsymbol{\vartheta}}$ is the unit vector normal to $\hat{\boldsymbol{r}}$ in the orbital plane (positive in the direction of the grain's motion), $\varphi=\arccos (\boldsymbol{w} \cdot \hat{\boldsymbol{r}}) / w$ is the angle that the average solar wind flow direction forms with the grain's velocity. The first part of Eq. (9) is the sum of radiation pressure force $\beta_{r} f_{g} \hat{\boldsymbol{r}}$ with the corpuscular pressure force $\beta_{w} f_{g} \hat{\boldsymbol{w}}=f_{w} \boldsymbol{w} / u$ split into its radial and transverse component. The latter part of Eq. (9) is the sum of the classical PR drag with the solar wind drag (Marzari \& Vanzani 1994).

The adopted numerical algorithm for solving the equations of motion is the RA15 version of the RADAU integrator (Everhart 1985). 


\section{Initial conditions for asteroidal dust and cometary dust particles}

To compute the flux of dust particles released in the main belt through asteroidal collisions and reaching the Earth and Mercury, we adopted an initial distribution of orbital elements with the following characteristics (Borin et al. 2009; Marzari et al. 1996; Dohnanyi 1969). The initial semimajor axis is randomly selected in between 2.1 and $3.3 \mathrm{AU}$, the initial eccentricity varies in the range $0.0-0.4$ and the inclination is in the range $0-20^{\circ}$. This choice reflects the typical orbital elements of the asteroid belt (Gradie et al. 1989; Zappalá \& Cellino 1993; Zappalá et al. 1994; Milani \& Knezevic 1994). We assume a grain density of $2.5 \mathrm{~g} \mathrm{~cm}^{-3}$, which is a reasonable value for dust particles coming from the main belt asteroids (Grün et al. 1985). Spherical particles are considered in the approximation of Mie's theory for which $Q_{\mathrm{pr}}$, the dimensionless, radiation-pressure coefficient averaged over the solar spectrum, is 0.53 (Marzari \& Vanzani 1994; Mukai et al. 1982).

For the JFC dust particles we considered orbits with an initial semimajor axis in a range between 2.5 and 26.7 AU with the majority of dust grains in the range between 2.5 and $8 \mathrm{AU}$, eccentricity in a range interval between 0.3 and 0.9 , and orbit inclination in the range between $0-81^{\circ}$ with a large concentration of particles in the range between $0-45^{\circ}$. In this case, we assume a density of $\varrho=1.0 \frac{\mathrm{g}}{\mathrm{cm}^{3}}$, the radiation pressure coefficient $Q_{\mathrm{pr}}=0.53$, following the asteroids grain simulations (Marzari \& Vanzani 1994; Mukai et al. 1982). The initial orbital elements of comets are taken from Nesvorny et al. (2010) and Levison \& Duncan (1997), who followed the evolution of bodies originating in the Kuiper Belt as they are scattered by planets and evolve into the inner solar system. In their input list of source objects, Nesvorny et al. (2010) consider a JFC to become active at the time when the perihelion distance, q first drops below 2.5 AU in the Levison \& Duncan (1997) simulations. The orbital distribution of visible JFCs obtained with this model by Levison \& Duncan (1997) well approximates the observed distribution.

Defining $t_{\mathrm{flt}}=12000 \mathrm{yr}$ as the fading lifetime of JFCs, which is the time range between their first and last appearance (Levison \& Duncan 1997; Nesvorny et al. 2010), we considered both the initial time $t=0$ and the final time $(t<12000)$ for each comet with perihelion distance $q<2.5 \mathrm{AU}$. However, the outcome in terms of close encounters and impact speed was not significantly different, so we used the set with the final time. In Fig. 1 we illustrate the orbital distributions of JFC comets at the final time. This orbital distribution is used for the initial trajectories of the dust particles in our numerical integrations. We take particles with radii of 10,50 , and $100 \mu \mathrm{m}$ into account.

While for asteroidal dust particles the initial mean anomaly is randomly sampled between $0^{\circ}$ and $360^{\circ}$, for JFC grains the particles are started at the perihelion when the mean anomaly $M=0$. This second sampling procedure for the mean anomaly is dictated by the relation between dust emission and cometary activity. Dust emission starts with comet outgassing, which has its peak at the perihelion where the comet is closer to the Sun. It is then reasonable to assume that dust reaching the Earth and Mercury was mostly produced during the perihelion passage.

\section{Estimates of the impact efficiency of grains on Mercury}

We compute the dynamical evolution of cometary and asteroidal dust grains until they cross the orbit of Mercury. We record every time a dust particle falls within ten times the sphere of influence of the planet as a close encounter, then we withdraw the particle
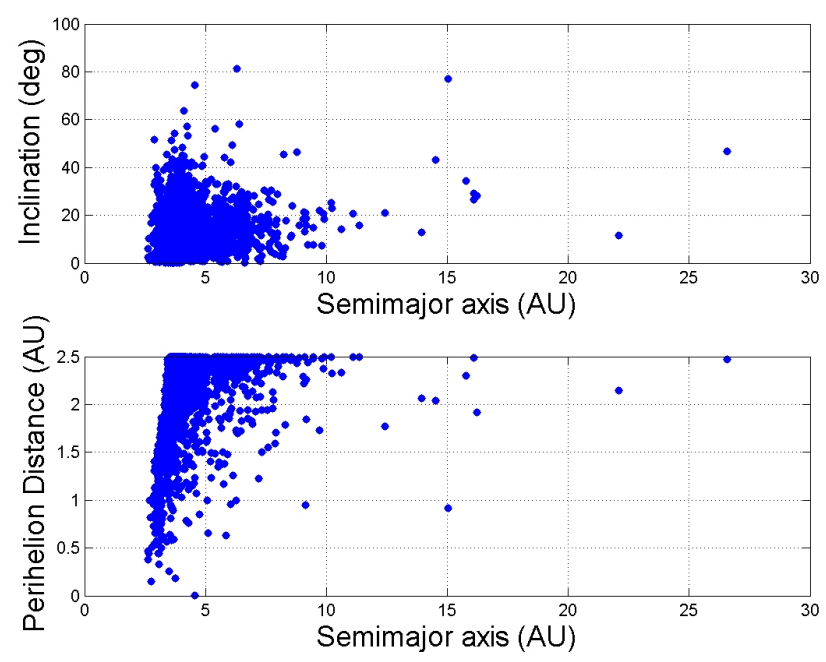

Fig. 1. Initial orbital distribution of JFCs. Figures show the inclination and perihelion distance as function of the semimajor axis.

from the sample (this is the same method used in Borin et al. 2009). During its evolution a grain may have close encounters with other planets, but in this case we do not eliminate the grain from the sample since this is part of its dynamical evolution. At the end of the run we know how many particles approached the planet with respect to those that did not have close encounters and flew inside. By comparing the fluxes of particles encountering the planet, we can evaluate the relative efficiency of the two sources of dust grains impacting Mercury, i.e. asteroids or JFCs. We run the same model for the Earth to estimate the two fluxes in a different dynamical environment. It is manifestly impossible to numerically integrate a number of particles large enough to record actual impacts. As a consequence, this methodology is the only feasible way to estimate the relative impact flux on Mercury from different sources and to compare it to that on the Earth. The outcomes of the four different numerical simulations, two for the Earth (asteroidal or cometary source) and two for Mercury (same sources), are summarised in Table 1. The simulations for Mercury, and, in particular, those for the asteroidal dust grains, required more time since the particles have to evolve past Mars, Earth, and Venus before reaching Mercury.

The percentages reported in Table 1 are divided into two groups and computed for different particle sizes. The two columns under the title "Asteroids" give the fraction of particles of asteroidal origin entering ten times the sphere of influence of Mercury and the Earth in the two runs, respectively. While $n_{\mathrm{A}}^{\mathrm{E}}$ is the number of particles encountering the Earth in the run that only considers the close approaches with the Earth, $n_{\mathrm{A}}^{\mathrm{M}}$ is the number of particles encountering Mercury in the run in which the particles are allowed to evolve inside Mercury's orbit. The percentage $p_{\mathrm{A}}$ of asteroidal grains from the same initial source impacting either Earth or Mercury is $p_{\mathrm{A}}^{\mathrm{E}}=n_{\mathrm{A}}^{\mathrm{E}} /\left(n_{\mathrm{A}}^{\mathrm{E}}+n_{\mathrm{A}}^{\mathrm{M}}\right)$ and $p_{\mathrm{A}}^{\mathrm{M}}=n_{\mathrm{A}}^{\mathrm{M}} /\left(n_{\mathrm{A}}^{\mathrm{E}}+n_{\mathrm{A}}^{\mathrm{M}}\right)$, respectively. The same applies to grains of cometary origin. In this computation, we assume that the number of dust grains that impact the terrestrial planets is not a significant fraction of the whole dust grain population moving towards the Sun. This allows us to separate the computation of encounters with the Earth and Mercury in two separate runs with the same initial conditions.

Table 1 shows that the number of impacts on Mercury for grains of asteroidal origin is higher compared to that on Earth. This is possibly a dynamical effect related to the increasing 
Table 1. Percentage of particles arriving on Earth and Mercury considering cometary and asteroidal sources.

\begin{tabular}{c|c|c|c|c}
\hline \hline \multirow{2}{*}{ Particle's size $(\mu \mathrm{m})$} & \multicolumn{2}{|c|}{ Asteroids } & \multicolumn{2}{c}{ Comets } \\
\cline { 2 - 5 } & Earth & Mercury & Earth & Mercury \\
\hline 10 & $40 \%$ & $60 \%$ & $53 \%$ & $47 \%$ \\
50 & $42 \%$ & $58 \%$ & $61 \%$ & $39 \%$ \\
100 & $43 \%$ & $57 \%$ & $68 \%$ & $32 \%$ \\
\hline
\end{tabular}
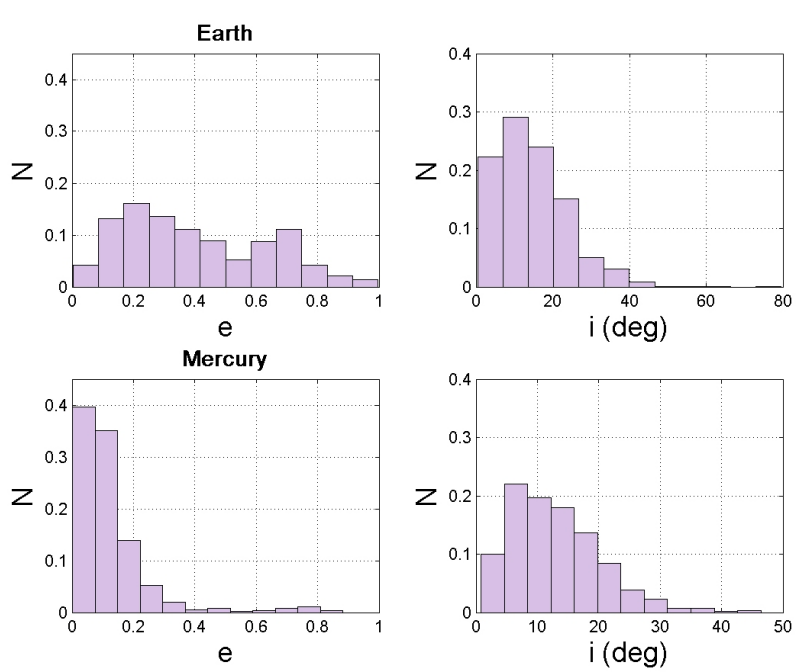

Fig. 2. Eccentricity and inclination of cometary dust particles at Earth and Mercury for particles of $50 \mu \mathrm{m}$.

density of the grains as they approach Mercury because of the smaller semimajor axis.

For cometary dust grains the situation is reversed. Compared to Mercury, the Earth is much more efficient in capturing particles within its sphere of influence, therefore, the number of impacts is expected to be lower on Mercury. According to Table 1, this effect is more pronounced in larger dust grains, and in effect there is a factor two of reduction in the number of encounters with Mercury for $100 \mu \mathrm{m}$ size particles. In Fig. 2 the distribution of the orbital elements of $50 \mu \mathrm{m}$ cometary grains is shown at the step before they have a close encounter with the planet. The eccentricities and inclinations of the dust grains as they reach Mercury are lower and this would possibly increase the probability of impact. However, as shown in Fig. 1 the initial orbits of the dust grains do not immediately cross Mercury's orbit. During the time that it takes the grains to move close to the planet under the effect of the PR-drag, a significant fraction of particles, more than $60 \%$ of our initial sample depending on their size, is ejected out of the system by close encounters with the other planets. Cometary dust particles are on high eccentric orbit from the start and, as a consequence, they are more easily thrown out by close approaches. This explains the trend observed as a function of the particle size. Smaller particles migrate inside faster and have a higher chance of reaching Mercury than larger grains, which are subject to close encounters with other planets for a longer time. In conclusion, dust particles of asteroidal origin are expected to be more relevant in the flux of grains impacting Mercury compared to cometary grains. This is the case at least when compared to what happens on Earth.

\section{Comparison between impact velocities for grains with different origins}

A by-product of our calculations of dust particle orbital evolution is a robust estimate of the impact velocity at Mercury.
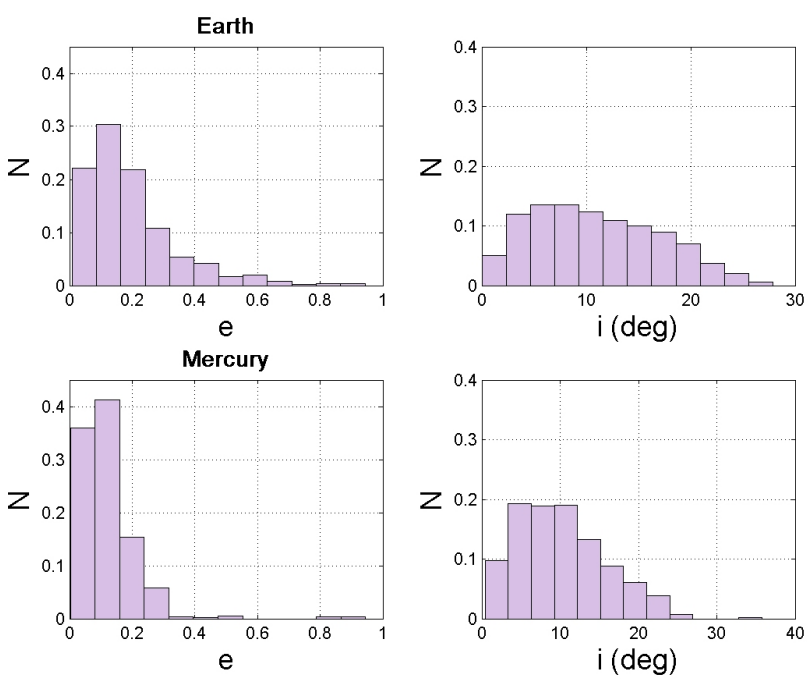

Fig. 3. Eccentricity and inclination of asteroidal dust particles at Earth and Mercury for particles of $50 \mu \mathrm{m}$.

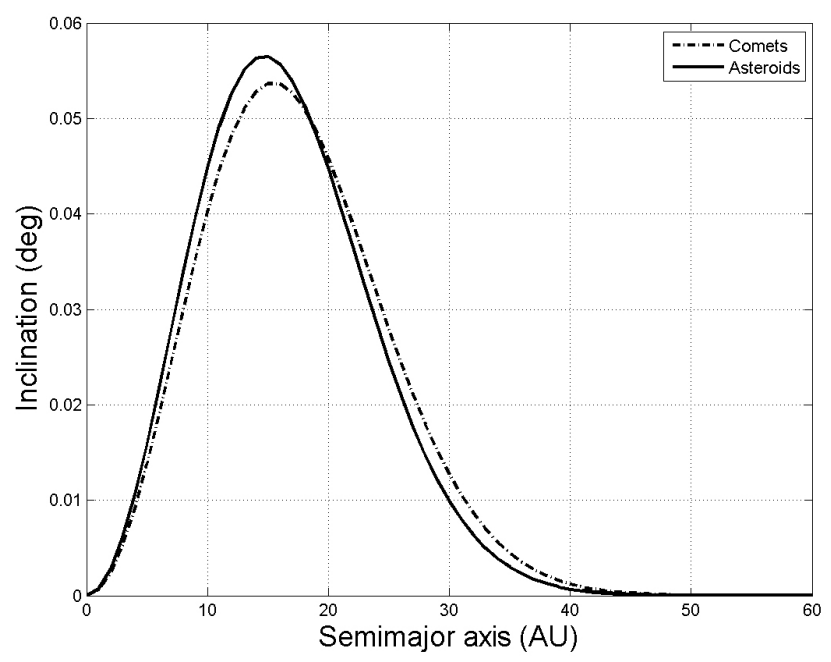

Fig. 4. Velocity distribution of asteroidal and cometary dust particles at Mercury.

Figure 4 illustrates the velocity distribution of dust particles when they encounter the planet. For asteroidal dust particles the mean velocity is $16.81 \mathrm{~km} \mathrm{~s}^{-1}$, while the cometary component has a mean velocity value of $17.59 \mathrm{~km} \mathrm{~s}^{-1}$. Figures 2 and 3 show the distribution of eccentricity and inclination of cometary and asteroidal dust grains at Earth and Mercury for particles of $50 \mu \mathrm{m}$ before the encounter. As expected, the eccentricity at Mercury shifts to lower values than Earth because of the PR drag. The shift is comparable for both cometary and asteroidal grains and there is little difference in the two distributions prior to the encounters. The inclination distribution is instead broader for cometary dust as a result of the initial distribution and this explains the higher relative velocity between the grains and the planet. The asteroidal dust particles are stirred in inclination by encounters with the planets and temporary resonance trapping, but the effect is not comparable to the initial inclination distribution of JFCs. 


\section{Conclusions}

We have used a dynamical model to estimate the flux of dust grains of cometary origin on Mercury. We calculate the orbital evolution of the grains under gravitational and non-gravitational forces and evaluate the efficiency of delivery on the planet. Our results show that the impact efficiency of particles from JFCs is reduced when moving from the Earth to Mercury by almost a factor two, depending on the grain size. The dynamical evolution of the dust produced in the asteroid belt increases the impacting flux from the Earth to Mercury since the inside migration induced by the PR drag causes an increase in the local dust density. On the other hand, cometary dust particles may be ejected out from the system before reaching Mercury's orbit and this leads to a decrease of cometary dust flux compared to that on Earth.

If we assume that the flux of grains impacting Earth is a mixture of asteroidal and cometary grains in different proportions, than we would expect that on Mercury the asteroidal component is reinforced with respect to the cometary component. According to Wiegert et al. (2009) and Nesvorny et al. (2010), the flux on Earth is dominated by cometary grains but on Mercury the situation might be more balanced.

This work could have interesting implications for the computation of atoms released in the exosphere of Mercury (Cintala 1992; Cremonese et al. 2005). The sources of Mercury's tenuous exosphere have been hypothesised to be photon-stimulated desorption, vaporisation by impact of meteoroids and micrometeoroids, and sputtering by the solar wind (Killen et al. 2007; Domingue et al. 2007). Mercury's surface is constantly bombarded by dust particles from the interplanetary medium that mix the surface regolith and create a vapor cloud at high energy that is representative of the surface composition. The amount of material produced by impact vaporisation depends on the impactor and surface compositions and the micrometeoroid flux to the surface. The ratio between the volume of melt and volume of vapor depends on the impact velocity as well as other impactor, surface, and impact-related parameters. At the moment, the impact vaporisation rate at Mercury is highly uncertain because of uncertainties in the density and velocity distribution of the interplanetary dust and the vapor production rate of the impacting dust particles (Burger et al. 2010). Recent works suggest that the enhancement in $\mathrm{Ca}$ at $25^{\circ} \pm 5^{\circ}$ after perihelion can be attributed to the crossing of Mercury's orbital plane and a Comet 2P/Encke stream (Killen \& Hahn 2015; Christou et al. 2015). Also, Sun-grazing comets should be further investigated as a possible source (Sprague et al. 2007). With this work, we intend to distinguish between the potential fluxes due to cometary and asteroidal sources to better understand the contribution of the two components.

Acknowledgements. This activity has been realised under the BepiColombo Agenzia Spaziale Italiana (ASI) contract to the Istituto Nazionale di Astrofisica (INAF) no I/022/10/0.

\section{References}

A'Hearn, M. F., Millis, R. C., Scheilder, D. O., Osip, D. J., \& Birch, P. V. 1995, Icarus, 118, 223

Borin, P., Cremonese, G., Marzari, F., Bruno, M., Marchi, S. et al. 2009, A\&A, 503, 259

Burger, K., Rosemary, M., Vervack, R. J., et al. 2010, Icarus, 209, 63

Burns, J. A., Lamy, P. L., Soter, S., et al. 1979, Icarus, 40, 1

Christou, A. A., Killen, R. M., Burger, M. H., et al. 2015, Geophys. Res. Lett., 42,7311

Cintala, M. J. 1992, J. Geophys. Res., 97, 947

Cremonese, G., Bruno, M., Mangano, V., Marchi, S., \& Milillo, A. 2005, Icarus, 177,122

Dermott, S. F., Nicholson, P. D., Burns, J. A., \& Houck, J. R. 1984, Nature, 312 505

Dermott, S. F., Durda, D. D., Grogan, K., \& Kehoe, T. J. J. 2002, Asteroidal dust, in Asteroids III, eds. W. F. Bottke, A. Cellino, P. Paolicchi, \& Binzel (Arizona University Press)

Dohnanyi, J. S. 1969, J. Geophys. Res., 74, 2531

Domingue, D. L., Koehn, P. L., Killen, R. M. et al. 2007, Space Sci. Rev., 131, 161

Everhart, E. 1985, Proc. IAU Colloq. 83, 115, 185

Fernández, Y. R. 2009, Planet. Space Sci., 57, 1218

Flynn, G. J. 2002, Extraterrestrial Dust in the Near-Earth Environment, Meteors in the Earth's atmosphere, eds. E. Murad, \& I. P. Williams (Cambridge University Press)

Fulle, M. 2004, Motion of cometary dust, Comets II, eds. M. C. Festou, H. U. Keller, \& H. A. Weaver (Tucson: University of Arizona Press), 745, 565

Gradie, J. C., Chapman, C. R., \& Tedesco, E. F. 1989, Distribution of Taxonomic Classes and the Compositional Structure of the Asteroid Belt, Asteroids II (Tucson: University of Arizona Press), Proc. Conf., 316

Grün, E., Zook, H. A., Fechtig, H., Giese, R. H., et al. 1985, Icarus, 62, 244

Ipatov, S. I., \& Mather, J. C. 2005, Migration of dust particles to the terrestrial planets, Proc. Dust in Planetary Systems, Kauai, Hawaii

Ipatov, S. I., \& Mather, J. C., 2006, Adv. Space Res., 37, 126

Jackson, A. A., \& Zook, H. A. 1992, Icarus, 97, 70

Killen, R.M., Cremonese, G., Lammer, H., et al. 2007, Space Sci. Rev., 132, 433Ü

Killen, R. M., \& Hahn, J.M. 2015, Icarus, 250, 230

Levison, H. F., \& Duncan, M. J. 1997, Icarus, 127, 13

Love, S. G., \& Brownlee, D. E. 1993, Science, 262, 550

Mann, I. 2004, Space Sci. Rev, 110, 269

Mann, I., \& Grün, E. 1995, Planet. Space Sci., 43, 827

Mann, I., Grün, E., Wilck, M., et al. 1996, Icarus, 120, 399

Marzari, F., \& Vanzani, V. 1994, A\&A, 283, 275.

Marzari, F., Scholl, H., Farinella, P., et al. 1996, Icarus, 119, 192

Milani, A., \& Knezevic, Z. 1994, Icarus, 107, 219

Mukai, T., \& Yamamoto, T. 1982, A\&A, 107, 97

Nesvorny, D., Jenniskens, P., Levison, H. F., et al. 2010, ApJ, 713, 816

O'Brien, D. P., \& Greenberg, R. 2003, Icarus, 164, 334

Sprague, A., Warell, J., Cremonese, G., et al. 2007, Space Sci. Rev., 132, 399

Sykes, M. V., \& Greenberg, R. 1986, Icarus, 65, 51

Sykes, M. V., Cellino, A., Farinella, P., Milani, A. et al. 2004, The Interplanetary Dust Complex and Comets, Comets II (Tucson: University of Arizona Press), 677

Zappalá, V., \& Cellino, A. 1993, Proc. 160th International Astronomical Union, held in Belgirate, Italy

Zappalá, Cellino, A., Farinella, P., \& Milani, A. 1994, ApJ, 107, 2

Zolensky, M. E., \& Warren, J. L. 1994, Collection and curation of interplanetary dust particles recovered from the stratosphere, Lunar and Planetary Inst., Workshop on the Analysis of Interplanetary Dust Particles, 56

Weissman, P. R. 2007, Proc. IAU Symp. 236

Wiegert, P. 2009, Icarus, 201, 295

Wyatt, S. P., \& Whipple, F.L. 1950, ApJ, 111, 134 